\title{
Comparing theoretical and practical solution of the first order first degree ordinary differential equation of population model
}

\begin{abstract}
Population dynamics is the branch of mathematics that studies the size and age composition of populations as dynamical systems, the biological and environmental processes driving them such as birth and death rates and by immigration and emigration. In this paper, we are discussed how to read mathematical models and how to analyze them with the ultimate aim that we can critically judge the assumptions and the contributions of such models whenever we encounter them in your future biological research. Mathematical models are used in all areas of biology. All models in this paper are formulated in ordinary differential equations (ODEs). These will be analyzed by computing steady states. We developed the differential equations by ourselves following a simple graphical procedure, depicting each biological process separately. Experience with an approach for writing models will help us to evaluate models proposed by others.
\end{abstract}

Volume I Issue I - 2018

\author{
Abdullah Bin Masud, Foyez Ahmed \\ Department of Computer Science \& Engineering, Shanto- \\ Mariam University of Creative Technology, India \\ Correspondence: Abdullah Bin Masud, Department of \\ Computer Science \& Engineering, Shanto-Mariam University of \\ Creative Technology, Dhaka-1230, Bangladesh, India, \\ Email masud05math@gmail.com
}

Received: January 25, 2018 | Published: February 21, 2018

Keywords: general equation of population growth, logistic equation, logistic, model for given data; solution of logistic model, comparing logistic model with actual data

\section{Introduction}

In 1798, English economist Thomas Malthus was stated that population would grow at a geometric rate while the food supply grows at an arithmetic rate. The theory has been seen as flawed because of the limited factors observed when he developed the Law. It does not include factors, such as technology, disease, poverty, international conflict and natural disasters.

Malthusian models have the form $P(t)=P_{0} e^{k t}$ where $\mathrm{P}_{0}$ is the initial number of population, $\mathrm{k}$ is population growth rate (Malthusian parameter) and $t$ is the time. Sometimes this model is called simple exponential growth model.

\section{General equation of population growth}

The rate of change of quantity $=$ the rate of births - The rate of deaths.

Suppose $P(t)$ is the population, $\alpha$ is the per capital births rate and $\beta$ is the per capital number of deaths population.

$$
\begin{aligned}
\frac{d P(t)}{d t} & =\alpha P(t)-\beta P(t) \\
& =P(t)(\alpha-\beta) \\
& =P(t) K \quad \text { where } K=\alpha-\beta
\end{aligned}
$$

This is the first order first degree ordinary differential equation. ${ }^{1}$ The solution of (1) is $P(t)=c e^{k t}$. If $t=0, P=P_{0} \quad \therefore P_{0}=C$ and $P=P_{0} e^{K t}$ Birth rate is constant and death rate is linearly increasing

$$
\begin{aligned}
& \text { If } \quad \alpha=\alpha_{0} \text { and } \beta=\beta_{0}+\beta_{1} P(t) \text { then we have } \\
& \frac{d P}{d t}=\alpha_{0} P(t)-\left\{\beta_{0}+\beta_{1} P(t)\right\} P(t)
\end{aligned}
$$

$$
=\left(\alpha_{0}+\beta_{0}\right) P(t)-\beta_{1} P^{2}(t)
$$

Birth rate constant and death rate is exponentially increasing

$$
\text { If } \beta=\beta_{1} e^{k t} \text { and } \alpha=\alpha_{0} \text { then } \frac{d p}{d t}=\alpha_{0} P(t)-\beta_{1} e^{k t} P(t)
$$

Birth rate constant and death rate is sine function

If $\beta=\beta_{1} \sin t$ and $\alpha=\alpha_{0}$ then $\frac{d P}{d t}=\alpha_{0} P(t)-\beta_{1} \operatorname{Sin}(t) P(t)$

Death rate constant and birth rate linearly increasing

If $\alpha=\alpha_{0}+\alpha_{1} P(t)$ and $\beta=\beta$ then $\frac{d P(t)}{d t}=\left(\alpha_{0}+\alpha_{1} P(t)\right) P(t)-\beta P(t)$

Death rate constant and birth rate exponentially increasing

$$
\text { If } \alpha=\alpha_{1} e^{k t} \text { and } \beta=\beta_{0} \text { then } \frac{d P(t)}{d t}=\alpha_{1} e^{k t} P(t)-\beta_{0} P(t)
$$

Death rate and birth rate are linearly increasing

$$
\text { If } \alpha=\alpha_{0}+\alpha_{1} P(t) \text { and } \beta=\beta_{0}+\beta_{1} P(t) \text { then }
$$

$$
\begin{aligned}
\frac{d P(t)}{d t} & =\left(\alpha_{0}+\alpha_{1} P(t)\right) P-\left(\beta_{0}+\beta_{1} P\right) P \\
& =\left(\alpha_{0}-\beta_{0}\right) P+\left(\alpha_{1}-\beta_{1}\right) P^{2}
\end{aligned}
$$

Logistic equation: In real population growth is not always unlimited but may have an upper limit $\mathrm{L}$ where population can no longer 
be sustained as time increase. The logistic ODE is

$$
\frac{d P}{d t}=K P\left(1-\frac{P}{L}\right)
$$

$$
\begin{aligned}
& P(t+1)-P(t)=K P\left(1-\frac{P}{L}\right) \\
& \Rightarrow \frac{\Delta P}{P}=K\left(1-\frac{P}{L}\right) . .
\end{aligned}
$$

Logistic model for given data: Since we have discrete data, then we describe the model using a difference equation. The equation (A) can be written as

The equation says that the ratio of $\Delta P$ to $\mathrm{P}$ is linear function of $\mathrm{P}$. First of all, let's consider the left hand side (LHS) of equation (2). We calculate the difference of the populations for two consecutive years,

\begin{tabular}{|c|c|c|c|c|c|c|c|c|}
\hline \multirow[t]{2}{*}{ Year } & \multicolumn{2}{|c|}{ Bangladesh } & \multicolumn{2}{|l|}{ India } & \multicolumn{2}{|l|}{ Pakistan } & \multicolumn{2}{|l|}{ Canada } \\
\hline & $P(t)$ & A & $P(t)$ & $\mathbf{a}$ & $P(t)$ & $\mathbf{a}$ & $P(t)$ & $\mathbf{a}$ \\
\hline 1950 & 2.859358 & 0.000425 & 2.982949 & 0.000264 & 2.858823 & 0.00023 & 2.79945 & 0.000566 \\
\hline 1951 & 2.860573 & 0.000402 & 2.983737 & $0: 00: 24$ & $2.85948 I$ & 0.000267 & 2.801036 & 0.000579 \\
\hline 1952 & 2.861723 & 0.000405 & 2.984562 & $0: 00: 25$ & 2.860244 & 0.0003 & 2.802656 & 0.00059 \\
\hline 1953 & 2.862882 & 0.000425 & 2.985416 & $0: 00: 25$ & 2.861101 & 0.000329 & 2.804309 & 0.000598 \\
\hline 1954 & 2.864098 & 0.000455 & 2.986294 & $0: 00: 26$ & 2.862042 & 0.000355 & 2.805987 & 0.000601 \\
\hline 1955 & $2.86540 \mathrm{I}$ & 0.000488 & 2.987191 & 0.000306 & 2.863058 & 0.000379 & 2.807674 & 0.000597 \\
\hline 1956 & 2.8668 & 0.000519 & 2.988106 & 0.000311 & $2.864 \mid 43$ & 0.0004 & 2.80935 & 0.000584 \\
\hline 1957 & 2.868287 & 0.000542 & 2.989036 & 0.000316 & 2.865289 & 0.00042 & 2.810992 & 0.000563 \\
\hline 1958 & 2.869843 & 0.000556 & 2.989982 & 0.000322 & $2.86649 \mid$ & 0.000437 & 2.812573 & 0.000532 \\
\hline 1959 & $2.87 \mid 439$ & 0.00056 & 2.990945 & 0.000328 & 2.867745 & 0.000454 & 2.814068 & 0.000494 \\
\hline 1960 & 2.873049 & 0.00056 & 2.991925 & 0.000333 & 2.869047 & 0.000468 & 2.815458 & 0.000454 \\
\hline $196 \mid$ & 2.874659 & 0.00056 & 2.992923 & 0.000338 & 2.87039 & 0.000481 & 2.816737 & 0.000418 \\
\hline 1962 & 2.876269 & 0.000567 & 2.993935 & 0.000342 & 2.87177 & 0.000491 & 2.817913 & 0.000391 \\
\hline 1963 & 2.877902 & 0.000584 & 2.99496 & 0.000344 & $2.873|8|$ & 0.000499 & 2.819015 & 0.000377 \\
\hline 1964 & 2.879585 & 0.000604 & 2.995992 & 0.000346 & 2.874615 & 0.000505 & 2.820079 & 0.000372 \\
\hline 1965 & 2.881326 & 0.00063 & 2.997029 & 0.000347 & 2.876068 & 0.000512 & 2.821129 & 0.000369 \\
\hline 1966 & $2.883 \mid 43$ & 0.000644 & 2.998069 & 0.000348 & 2.877539 & 0.000517 & 2.82217 & 0.000363 \\
\hline 1967 & $2.88500 \mathrm{I}$ & 0.000625 & 2.999113 & 0.000352 & 2.879027 & 0.000521 & 2.823194 & 0.000358 \\
\hline 1968 & 2.886806 & 0.000567 & 3.000168 & 0.000358 & 2.880528 & $9.55 \mathrm{E}-09$ & 2.824204 & 0.000352 \\
\hline 1969 & 2.888444 & 0.000487 & 3.001242 & 0.000366 & 2.880528 & 0.001048 & 2.825197 & 0.000345 \\
\hline 1970 & 2.889852 & 0.0004 & 3.002339 & 0.000374 & 2.883547 & 0.000525 & 2.826173 & 0.00034 \\
\hline |97| & 2.891009 & 0.000333 & 3.003461 & 0.00038 & 2.885062 & 0.000528 & $2.827 \mid 35$ & 0.000335 \\
\hline 1972 & 2.89197 & 0.000304 & 3.004603 & 0.000383 & 2.886585 & 0.000534 & 2.828082 & 0.000325 \\
\hline 1973 & 2.89285 & 0.000326 & 3.005755 & 0.000383 & $2.888 \mid 27$ & 0.000546 & 2.829 & 0.000308 \\
\hline 1974 & 2.893793 & 0.000382 & 3.006908 & 0.000381 & 2.889702 & 0.00056 & 2.829872 & 0.000288 \\
\hline 1975 & 2.894898 & 0.000446 & 3.008055 & 0.000378 & $2.89 \mid 321$ & 0.000573 & 2.830687 & 0.000268 \\
\hline 1976 & 2.896189 & 0.000495 & 3.009191 & 0.000376 & 2.892979 & 0.000585 & $2.83 \mid 444$ & 0.00025 \\
\hline 1977 & 2.897622 & 0.000526 & 3.010324 & 0.000372 & 2.89467 & 0.000596 & 2.832153 & 0.000236 \\
\hline 1978 & 2.899147 & 0.000533 & 3.011443 & 0.000374 & 2.896396 & 0.000607 & 2.832822 & 0.000226 \\
\hline 1979 & 2.900694 & 0.000524 & 3.01257 & 0.000375 & 2.898155 & 0.000617 & 2.833463 & 0.00022 \\
\hline 1980 & 2.902215 & 0.000512 & 3.013701 & 0.000377 & 2.899945 & 0.000626 & 2.834085 & 0.000213 \\
\hline 1981 & 2.903702 & 0.000505 & 3.014838 & 0.000377 & 2.901759 & 0.000631 & 2.834688 & 0.000207 \\
\hline 1982 & 2.905169 & 0.000534 & 3.015975 & 0.000375 & 2.903589 & 0.000631 & 2.835275 & 0.00021 \\
\hline 1983 & 2.90672 & 0.000466 & 3.017106 & $0.00037 \mid$ & 2.905421 & 0.000626 & $2.83587 \mid$ & 0.000223 \\
\hline 1984 & 2.908076 & 0.000502 & 3.018225 & 0.000365 & 2.90724 & 0.000617 & 2.836503 & 0.000242 \\
\hline 1985 & 2.909535 & 0.000503 & 3.019325 & 0.000358 & 2.909035 & 0.000608 & 2.837191 & 0.000264 \\
\hline 1986 & 2.911 & 0.000501 & 3.020407 & 0.000352 & 2.910804 & 0.000598 & $2.83794 \mid$ & 0.000282 \\
\hline
\end{tabular}
and then use those differences against the corresponding function

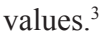

Citation: Masud AB, Ahmed F. Comparing theoretical and practical solution of the first order first degree ordinary differential equation of population model. Open Acc J Math Theor Phy. 20 I8; I(I):I I-17. DOI: 10.15406/oajmtp.2018.01.00003 


\begin{tabular}{|c|c|c|c|c|c|c|c|c|}
\hline \multirow[t]{2}{*}{ Year } & \multicolumn{2}{|c|}{ Bangladesh } & \multicolumn{2}{|l|}{ India } & \multicolumn{2}{|l|}{ Pakistan } & \multicolumn{2}{|l|}{ Canada } \\
\hline & $P(t)$ & A & $P(t)$ & $\mathbf{a}$ & $P(t)$ & $\mathbf{a}$ & $P(t)$ & $\mathbf{a}$ \\
\hline 1987 & 2.912459 & 0.000493 & 3.02147 & 0.000346 & 2.912544 & 0.000582 & $2.83874 I$ & 0.000291 \\
\hline 1988 & 2.913895 & 0.000478 & $3.0225 \mid 4$ & 0.000339 & 2.91424 & 0.000562 & 2.839566 & 0.000287 \\
\hline 1989 & 2.915288 & 0.000458 & 3.02354 & 0.000333 & 2.915877 & 0.000538 & 2.84038 & 0.000274 \\
\hline 1990 & 2.916624 & 0.000437 & 3.024548 & 0.000327 & 2.917446 & $0.0005 \mid 4$ & $2.84 I I 57$ & 0.000259 \\
\hline 1991 & 2.917898 & 0.000418 & 3.025537 & 0.000321 & 2.918945 & 0.000492 & 2.841894 & 0.000247 \\
\hline 1992 & 2.919117 & 0.000404 & 3.026507 & 0.000315 & 2.92038 & 0.000475 & 2.842597 & 0.000235 \\
\hline 1993 & 2.920298 & 0.000398 & 3.02746 & 0.00031 & 2.921767 & 0.000466 & 2.843265 & 0.000224 \\
\hline 1994 & 2.921459 & 0.000395 & 3.028401 & 0.000307 & 2.923128 & 0.000461 & $2.84390 \mathrm{I}$ & 0.000213 \\
\hline 1995 & $2.9226 \mid 4$ & 0.000393 & 3.029329 & 0.000303 & 2.924474 & 0.000458 & 2.844507 & 0.000202 \\
\hline 1996 & 2.923763 & 0.000388 & 3.030247 & 0.000299 & 2.925813 & 0.000453 & 2.845083 & 0.000192 \\
\hline 1997 & 2.924899 & 0.000381 & 3.031152 & 0.000294 & 2.927139 & 0.000444 & 2.84563 & 0.000186 \\
\hline 1998 & 2.926014 & 0.00037 & 3.032042 & 0.000288 & 2.928439 & 0.00043 & $2.84616 \mathrm{I}$ & 0.000185 \\
\hline 1999 & 2.927098 & 0.000495 & 3.032915 & 0.000281 & 2.929697 & 0.000412 & 2.846688 & 0.000188 \\
\hline 2000 & 2.928547 & 0.000205 & 3.033768 & 0.000275 & 2.930905 & 0.000395 & 2.847223 & 0.000191 \\
\hline 2001 & 2.929148 & 0.000331 & 3.034601 & 0.000268 & $2.93206 \mathrm{I}$ & 0.00038 & 2.847767 & 0.000194 \\
\hline 2002 & 2.930118 & 0.000314 & 3.035415 & 0.000262 & 2.933176 & I & 2.84832 & 0.000199 \\
\hline 2003 & 2.931039 & 0.000352 & 3.03621 & 0.000255 & 2.934264 & 0.000367 & 2.848887 & 0.000206 \\
\hline 2004 & $2.93207 \mid$ & $0.000 \mid 44$ & 3.036985 & 0.000249 & $2.93534 \mathrm{I}$ & 0.000356 & 2.849474 & 0.000214 \\
\hline 2005 & 2.932492 & $0.00037 \mid$ & $3.03774 \mid$ & 0.000243 & 2.936387 & 0.000381 & 2.850083 & 0.000222 \\
\hline 2006 & 2.933579 & 0.000152 & 3.03848 & 0.000237 & 2.937506 & 0.00037 & 2.850717 & 0.000229 \\
\hline 2007 & 2.934025 & 0.000204 & 3.039199 & 0.00023 & 2.938592 & 0.000371 & 2.85137 & 0.000233 \\
\hline 2008 & 2.934624 & 0.000201 & 3.0399 & 0.000223 & 2.939683 & 0.000374 & 2.852033 & 0.000231 \\
\hline 2009 & 2.935213 & 0.000205 & 3.040578 & 0.000216 & $2.94078 \mathrm{I}$ & 0.000376 & 2.852692 & 0.000226 \\
\hline 2010 & 2.935816 & 0.000212 & 3.041235 & 0.000209 & 2.941887 & 0.000378 & 2.853337 & 0.00022 \\
\hline 2011 & 2.936438 & 0.000217 & 3.04187 & 0.000202 & 2.942999 & 0.00038 & 2.853965 & 0.000214 \\
\hline 2012 & 2.937075 & 0.000219 & 3.042483 & 0.000196 & 2.944117 & 0.000379 & 2.854577 & 0.000209 \\
\hline 2013 & 2.93772 & 0.000219 & 3.04308 & 0.000192 & 2.945232 & 0.000375 & 2.855172 & 0.000203 \\
\hline 2014 & 2.938363 & 0.000216 & 3.043665 & 0.000189 & 2.946337 & 0.00037 & 2.855752 & 0.000198 \\
\hline 2015 & 2.938997 & 0.000213 & 3.044241 & 0.000187 & 2.947427 & 0.000364 & 2.856318 & 0.000193 \\
\hline 2016 & 2.939623 & 0.00021 & $3.0448 I$ & 0.000184 & 2.948499 & 0.000357 & 2.85687 & 0.000187 \\
\hline
\end{tabular}

Determining the value of $\mathrm{K}$ and $\mathrm{L}$ : In the Least Square Approximation graph, we know the equation for the line, which is,
Substituting the point $\mathrm{P}(1950)$ and $\mathrm{P}(1951)$ in (10) we have

$y=a+b x$ .(3)

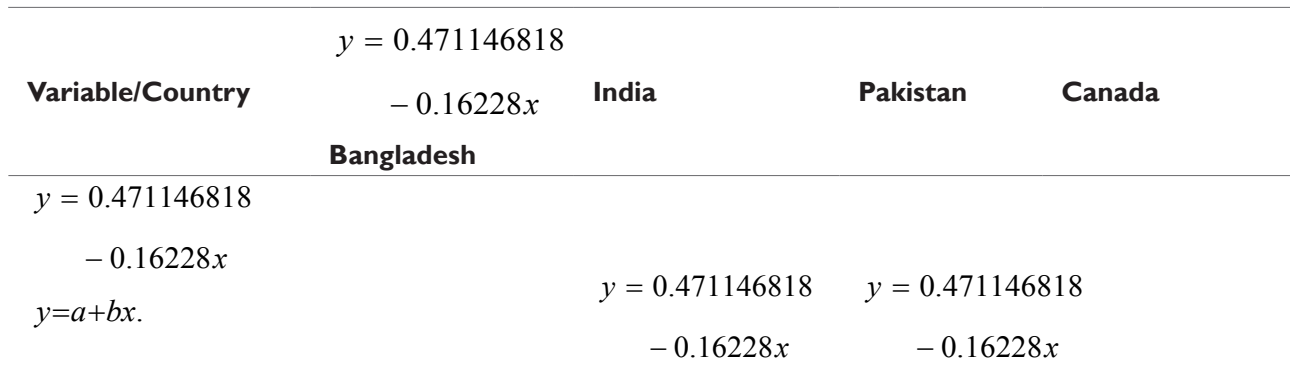

Citation: Masud AB,Ahmed F. Comparing theoretical and practical solution of the first order first degree ordinary differential equation of population model. Open Acc J Math Theor Phy. 20 I 8; I ( I): I I-I 7. DOI: I0.15406/oajmtp.2018.01.00003 


\begin{tabular}{lllll} 
Table Continued.. & & & & \\
$\mathrm{P}_{1}$ & 2.859358 & 2.982949 & 2.858823 & 2.79945 \\
$\mathrm{P}_{1}$ & 2.860576 & 2.983737 & $2.85948 \mathrm{I}$ & $67: 13: 29$ \\
$\mathrm{y}_{1}$ & 0.045499 & 0.033148 & 0.045835 & $0: 50: 01$ \\
$\mathrm{y}_{2}$ & 0.044285 & 0.032359 & 0.045177 & $0: 47: 44$ \\
\hline
\end{tabular}

Equation (2) can be written as

$K\left(1-P_{1} / L\right)=y_{1}$

and $K\left(1-P_{2} / L\right)=y_{2}$
Solving (3) and (4) we have $L=\frac{P_{1} y_{2}-P_{2} y_{1}}{y_{2}-y_{1}}$ and $K=\frac{y_{1}}{1-P_{1} / L}$

\begin{tabular}{lllll}
\hline Variable/ Country & Bangladesh & India & Pakistan & Canada \\
\hline L(Caring Capacity $)$ & 2.904878989 & 3.016041509 & 2.90468156 & 2.834196893 \\
Exp $(\operatorname{Exp}(\mathrm{L}))$ & 85415102.72 & 731302266 & 85107708.4 & 24562428.95 \\
K (Constant) & 2.903479866 & 3.021126376 & 2.90316821 & 2.833056521 \\
\hline
\end{tabular}

\section{Solution of logistic model}

Equation (1) is Bernoulli equation, ${ }^{4}$ we have

$\frac{d P}{d t}=K P\left(1-\frac{P}{L}\right)$

$\Rightarrow \frac{d P}{d t}=K P-\frac{K}{L} P^{2}$

$\Rightarrow \frac{d P}{d t}-K P=-\frac{K}{L} P^{2}$

$\Rightarrow-\frac{1}{P^{2}} \frac{d P}{d t}+\frac{K}{P}=\frac{K}{L}$

Put $\frac{1}{P}=V$

$\therefore-\frac{1}{P^{2}} \frac{d P}{d t}=\frac{d V}{d t}$

From (13) we have $\frac{d V}{d t}+K V=\frac{K}{L}$

Now this equation is exact. Hence integrating factor

$$
\begin{aligned}
& V \cdot e^{K t}=\int \frac{K}{L} e^{K t} d t \\
\Rightarrow & V \cdot e^{K t}=\frac{K e^{K t}}{L}+c \\
\Rightarrow & \frac{1}{P} e^{K t}=\frac{1}{L} e^{K t}+c \\
\Rightarrow & \frac{1}{P}=\frac{1}{L}+c e^{-K t} \\
\Rightarrow & P=\frac{L}{1+L c e^{-K t}} \ldots . . .
\end{aligned}
$$

If $t \rightarrow \infty$, then $\mathrm{P}=\mathrm{L}$.

\section{Comparing logistic model with actual data}

$\frac{d P}{d t}=P\left(K-\frac{P K}{L}\right)$

$\Rightarrow \frac{d p}{d t}=K P\left(\frac{L-P}{L}\right)$

$$
\begin{aligned}
I F & =e^{\int K d t} \\
& =e^{K t}
\end{aligned}
$$

\begin{tabular}{|c|c|c|c|c|c|c|c|c|c|}
\hline \multirow[t]{2}{*}{ Year } & \multirow[t]{2}{*}{ Time } & \multicolumn{2}{|l|}{ Bangladesh } & \multicolumn{2}{|l|}{ India } & \multicolumn{2}{|l|}{ Pakistan } & \multicolumn{2}{|l|}{ Canada } \\
\hline & & $\begin{array}{l}\text { Theoretical } \\
\text { Data }\end{array}$ & $\begin{array}{l}\text { Original } \\
\text { Data }\end{array}$ & $\begin{array}{l}\text { Theoretical } \\
\text { Data }\end{array}$ & $\begin{array}{l}\text { Original } \\
\text { Data }\end{array}$ & $\begin{array}{l}\text { Theoretical } \\
\text { Data }\end{array}$ & $\begin{array}{l}\text { Original } \\
\text { Data }\end{array}$ & $\begin{array}{l}\text { Theoretical } \\
\text { Data }\end{array}$ & $\begin{array}{l}\text { Original } \\
\text { Data }\end{array}$ \\
\hline 1950 & 0 & 0.564912 & 2.859358 & $0.54899 \mid$ & 2.982949 & $0.56569 \mid$ & 2.858823 & 0.525742 & 2.79945 \\
\hline $195 \mid$ & $\mathrm{I}$ & 0.338815 & 2.860573 & $2.47408 \mid$ & 2.983737 & 2.367713 & $2.85948 \mid$ & 2.252355 & 2.801036 \\
\hline
\end{tabular}

Hence the solution is

$\Rightarrow \frac{1}{K} \frac{L}{P(L-P)} d P=d t$

Integrating we have, $\frac{1}{K} \operatorname{Ln}\left(\frac{P}{L-P}\right)=t+c$

If $\mathrm{t}=0$ then find the value of $\mathrm{c}$

\begin{tabular}{lllll}
\hline Variable/ Country & Bangladesh & India & Pakistan & Canada \\
\hline L(Caring Capacity $)$ & 2.904878989 & 3.016041509 & 2.90468156 & 2.834196893 \\
$\operatorname{Exp}(\operatorname{Exp}(\mathrm{L}))$ & 85415102.72 & 731302266 & 85107708.4 & 24562428.95 \\
K (Constant) & 2.903479866 & 3.021126376 & 2.90316821 & 2.833056521 \\
\hline
\end{tabular}

Putting the values of $\mathrm{c}$ in (7), we have

Citation: Masud AB,Ahmed F. Comparing theoretical and practical solution of the first order first degree ordinary differential equation of population model. Open Acc J Math Theor Phy. 20 I8; I ( I):I I-I 7. DOI: I0.15406/oajmtp.20 I8.01.00003 
Table Continued.

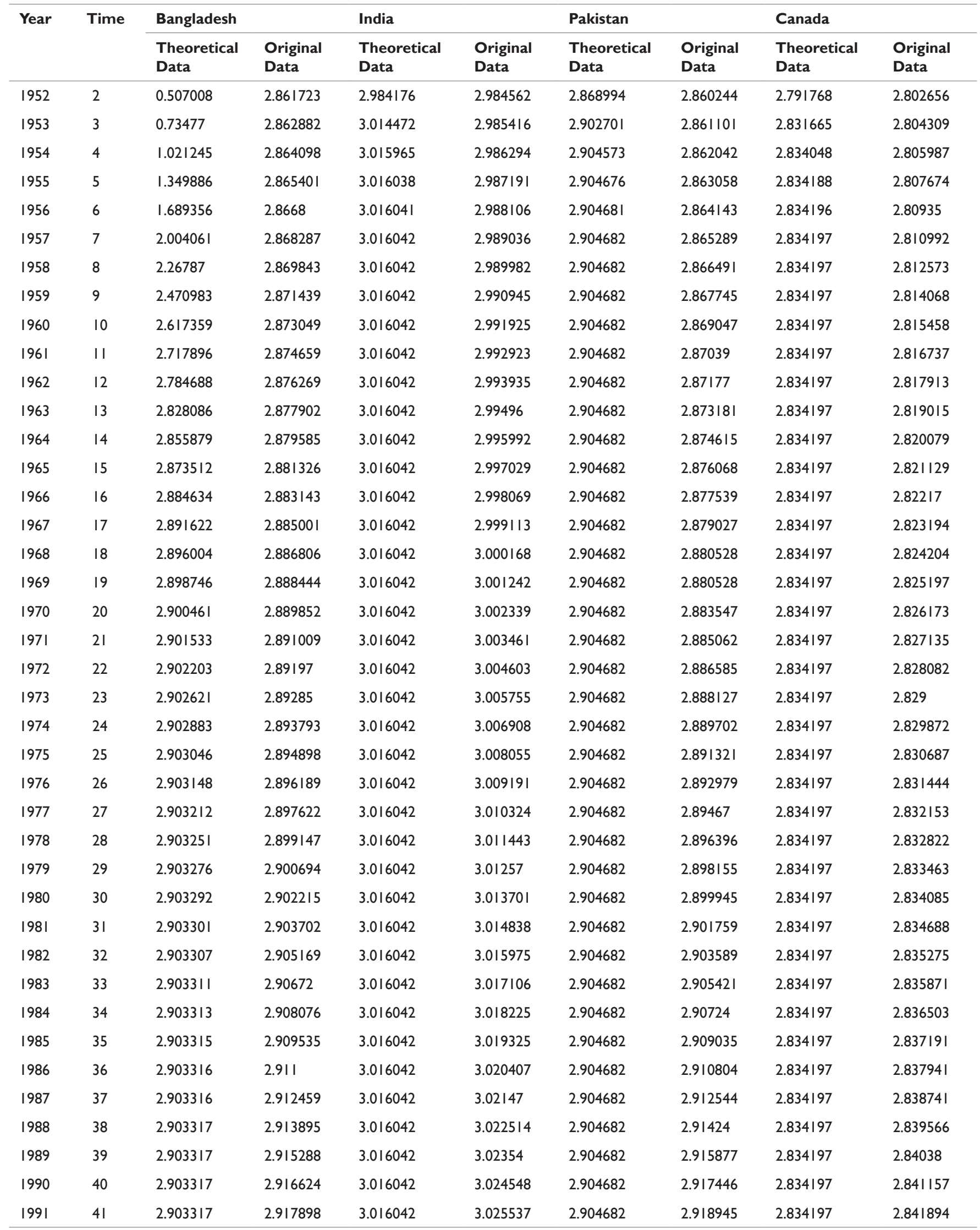

Citation: Masud AB,Ahmed F. Comparing theoretical and practical solution of the first order first degree ordinary differential equation of population model. Open Acc J Math Theor Phy. 20 I8; I ( I): I I-I 7. DOI: I0.15406/oajmtp.20 I8.01.00003 
Table Continued.

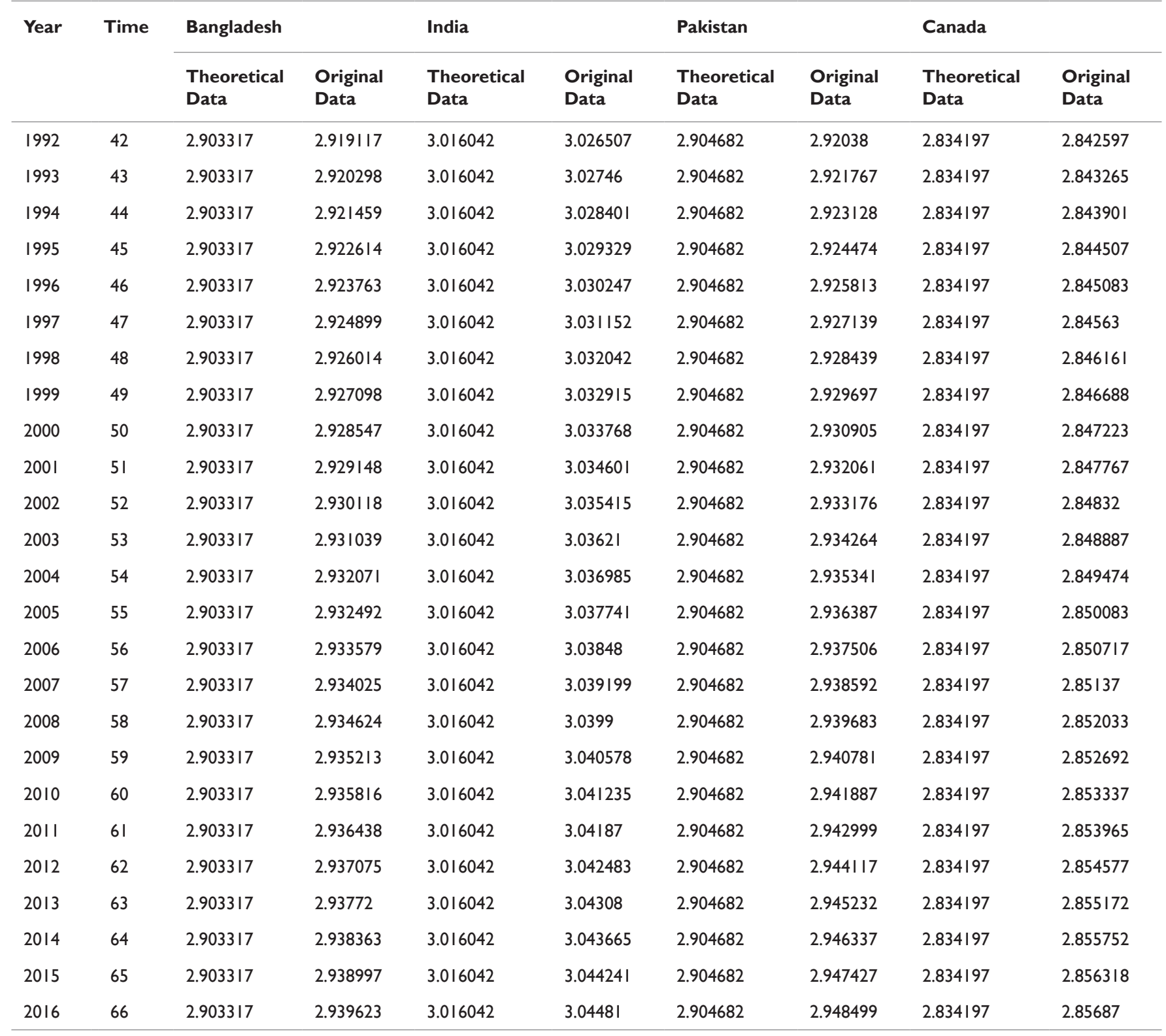

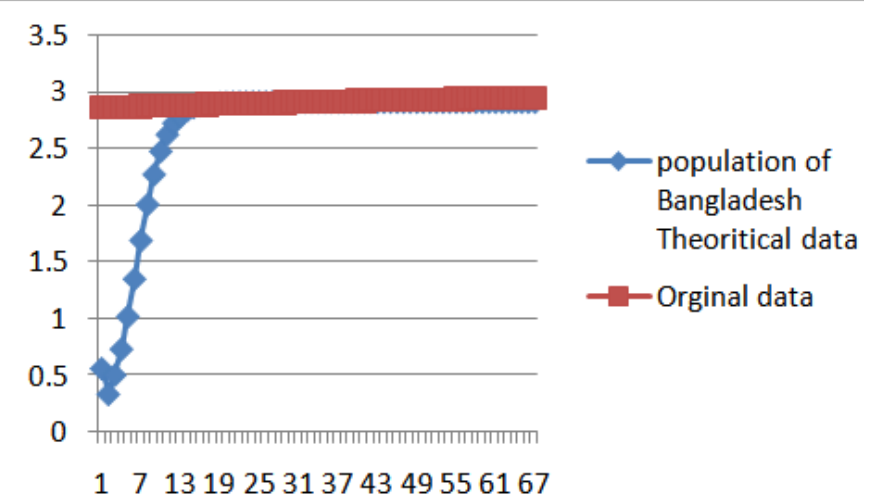

Figure I Comparing graph of theoretical data with original data of Bangladesh.

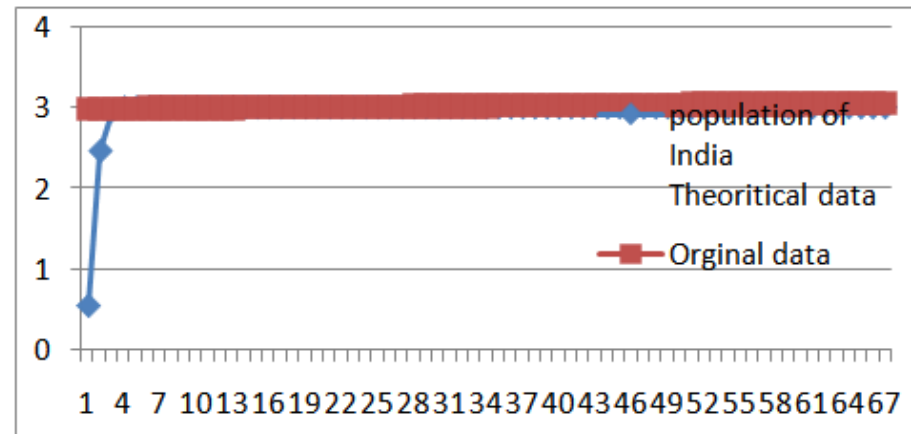

Figure 2 Comparing graph of theoretical data with original data of India.

Citation: Masud AB,Ahmed F. Comparing theoretical and practical solution of the first order first degree ordinary differential equation of population model. Open Acc J Math Theor Phy. 20 I8; I (I):I I-I7. DOI: I0.15406/oajmtp.20I8.0I.00003 


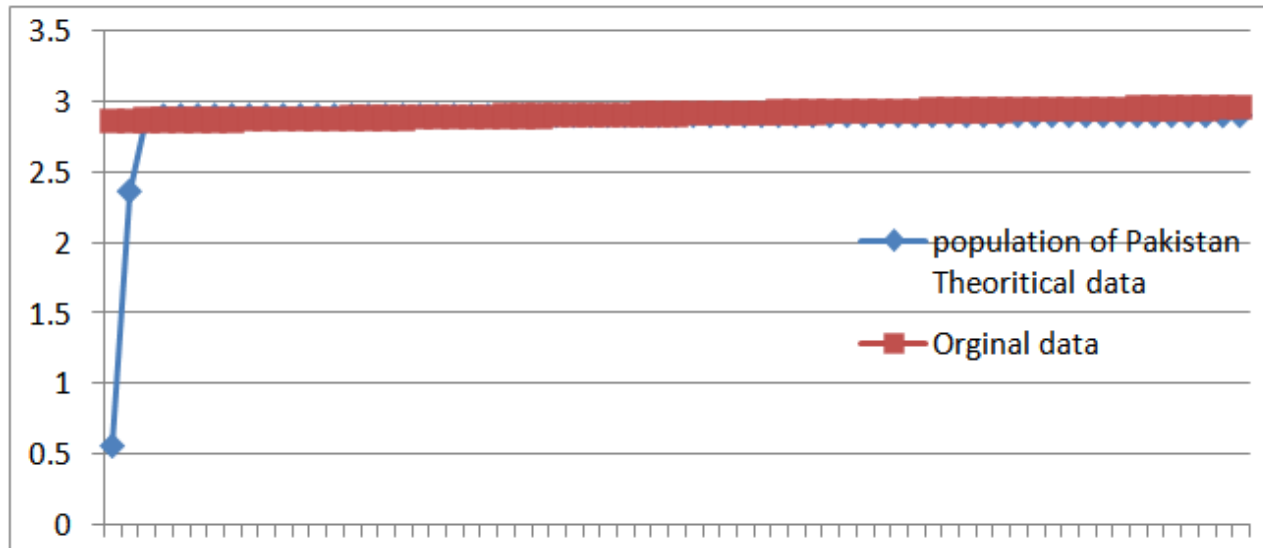

$\begin{array}{lllllllllllllllllllllll}1 & 4 & 7 & 10 & 13 & 16 & 19 & 22 & 25 & 28 & 31 & 34 & 37 & 40 & 43 & 46 & 49 & 52 & 55 & 58 & 61 & 64 & 67\end{array}$

Figure 3 Comparing graph of theoretical data with original data of Pakistan.

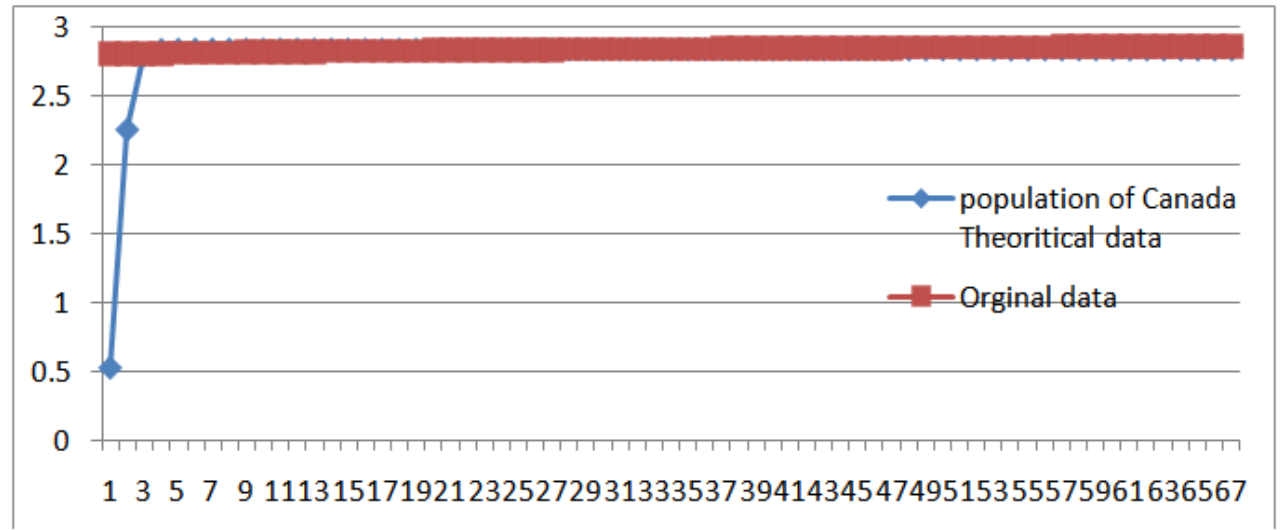

Figure 4 Comparing graph of theoretical data with original data of Canada.

\section{Conclusion}

The carrying capacity of Bangladesh is 85415102.72 but at this moment total number of population is 164827718 . It is the biggest problem. The government of Bangladesh needs to take necessary step otherwise socio economic system is breakdown. Every country of Subcontinent, the total population of these countries is grater twice of carrying capacity. In Canada, total number of population is greater the carrying capacity.

\section{Acknowledgements}

None.

\section{Conflict of interest}

The author declares no conflict of interest.

\section{References}

1. Dreyer TP. Modeling with Ordinary Differential Equations. USA: CRC Press; $1993.304 \mathrm{p}$

2. Kelley W, Peterson A. Theory of Differential Equations Classical and Qualitative. Springer; 2004.

3. Mooney DD, Swift RJ. A Course in Mathematical Modeling. UK: Cambridge University Press; $1999.431 \mathrm{p}$.

4. Zill DD. A First Course in Differential Equations. 1993. 